\title{
Multi-sensor-based Environmental Forecasting System for Smart Durian Farms in Tropical Regions
}

\author{
Ping-Huan Kuo, ${ }^{1,2}$ Ren-Jean Liou, ${ }^{3}$ Pongpon Nilaphruek, ${ }^{4}$ \\ Keeratiburt Kanchanasatian, ${ }^{5}$ Ting-Hao Chen, ${ }^{6}$ and Rong-Mao Lee ${ }^{6 *}$ \\ ${ }^{1}$ Department of Mechanical Engineering, National Chung Cheng University, \\ No. 168, Sec. 1, University Rd., Minhsiung Township, Chiayi County 621301, Taiwan \\ ${ }^{2}$ Advanced Institute of Manufacturing with High-tech Innovations (AIM-HI), National Chung Cheng University, \\ No. 168, Sec. 1, University Rd., Minhsiung Township, Chiayi County 621301, Taiwan \\ ${ }^{3}$ Department of Computer and Communication, National Pingtung University, \\ No. 4-18, Minsheng Rd., Pingtung City, Pingtung County 900391, Taiwan \\ ${ }^{4}$ Division of Big Data Management and Analytics, Rajamangala University of Technology Thanyaburi, \\ 39 Moo 1, Klong Luang Pathum Thani 12110, Thailand \\ ${ }^{5}$ Division of Computer Technology, Rajamangala University of Technology Thanyaburi, \\ 39 Moo 1, Klong Luang Pathum Thani 12110, Thailand \\ ${ }^{6}$ Department of Intelligent Robotics, National Pingtung University, \\ No. 4-18, Minsheng Rd., Pingtung City, Pingtung County 900391, Taiwan
}

(Received May 14, 2021; accepted August 20, 2021)

Keywords: smart farming, forecasting system, machine learning, CNN, SVM

Durians are among the most important fruit products in tropical countries. The environments of durians therefore must support a high yield to meet demand. Sunlight, temperature, and rainfall are all key variables, and any adverse factors will have a negative impact on production. We propose an environmental prediction system for a durian farm on the basis of the concept of the IoT. The system uses multiple machine learning algorithms to analyze collected environmental data and predict the next state of the environmental variables. From numerous experiments, our results show that the support vector machine (SVM) gives the best forecasts for temperature, whereas the convolutional neural network (CNN) performs best for predicting soil humidity. The results of this paper can provide farmers with real-time understanding of their farms and early warning of potential risks. The farm yield rates can hence be increased.

\section{Introduction}

Global warming has resulted in extreme weather and caused serious disasters on Earth. Agriculture has become a direct victim of global warming. Farmers must adapt traditional farming techniques such as irrigation, fertilizing, and harvesting to accommodate rapid weather changes. Building an intelligent system that can monitor and predict the variations of the environment will give farmers immediate understanding of their farm's status and the ability to react quickly.

*Corresponding author: e-mail: maxmou@mail.nptu.edu.tw https://doi.org/10.18494/SAM.2021.3580 
Durian trees are large and can grow as tall as $25 \mathrm{~m}$. Maintaining an appropriate soil moisture level promotes the growth of the fruit. Hence, irrigation is an important technique for growing durians. Good control of the growth environment will result in the production of high-quality fruit. However, traditionally the farming process has been solely based on experience. No environmental variables were measured, and hence no intelligent system could be built. The aim of this work is, in cooperation with farmers, to monitor, record, and predict growth variables in a durian farm by applying the concepts of IoT and smart farming. Machine learning techniques were utilized to train our system to accurately predict environmental variables.

Numerous applications have implemented the concepts of IoT and smart farming. Muangprathub et al. ${ }^{(1)}$ applied IoT techniques to collect data from environmental sensors and send reminders to farmers via a smart communication device. A remote irrigation system can be controlled by such smart devices. This technique, which was also applied to the cultivation of homegrown vegetables and limes, is one of the successful examples of smart farming. Sadowski and $\operatorname{Spachos}^{(2)}$ used sensors to acquire soil humidity. Information on the condition of the soil was then sent to farmers via Zigbee, WiFi, and LoraWAN in real time. The system utilized solar power, giving it the advantages of energy-saving and mobility, which are important issues in smart farming. A technique using a wireless sensor network was applied in Ref. 3 to analyze energy efficiency. Then, Torres et al. ${ }^{(4)}$ proposed a multilevel data fusion technique using a Kalman filter, decision trees (DTs), linear regression, and a support vector machine (SVM) to implement the tasks required by smart farming. The function of the evapotranspiration (the combined process of water surface evaporation, soil moisture evaporation, and plant transpiration) model generated using the SVM is very similar to that of the evapotranspiration reference model. Data visualization and cluster analysis are important tools to find effective technologies, as discussed in Ref. 5.

In Ref. 6, the importance of wireless networks to the smart farming IoT architecture was highlighted. For large farms, stability, speed, and efficiency are essential for building a wireless infrastructure. Hu et al. ${ }^{(7)}$ used nonorthogonal multiple access to increase the stability and efficiency of wireless communication. Malik et al. ${ }^{(8)}$ applied the concept of fog computing for sustainable intelligent agriculture, which demonstrated good performance in simulation results. Intelligent agriculture has many applications. In Ref. 9, image recognition was applied to crop detection; the system utilized deep learning and was validated satisfactorily. Sa et al. ${ }^{(10)}$ used a micro aerial vehicle to take aerial photographs of a farm. Deep learning was then applied to detect weeds to minimize damage to surrounding plants. The above studies achieved impressive results and have become well known. However, the applications of intelligent agriculture are mainly on a case-by-case basis.

This paper focuses on smart farming in a durian farm, which has seldom been discussed in previous works. Our proposed system collects real-time environmental data from various on-site sensors to build up a database. Then, a data model is created and analyzed using deep learning techniques. The deep network is trained using the records and then makes predictions according to the trends of the environmental data.

Several models based on machine learning have been developed for prediction. The multilayer perceptron (MLP), ${ }^{(11-13)}$ convolutional neural network $(\mathrm{CNN}),{ }^{(14-16)}$ long short-term 
memory (LSTM), ${ }^{(17-20)} \mathrm{SVM},{ }^{(21-24)}$ and random forest (RF) ${ }^{(25-28)}$ are among the most popular ones. We analyzed and compared these techniques to find the best fitting model for air temperature and soil moisture forecasts. The outcome can be used to facilitate the farming of durians.

The contributions of this paper are as follows. 1) The construction of a smart durian farm based on the IoT concept; 2) the debugging, cleaning, and organizing of environmental data to create an efficient database; 3 ) the synthesis of various farming models for machine learning; and 4) the analysis and comparison of experimental results to find the best prediction model for a durian farm.

This paper is organized as follows. Section 2 introduces the architecture of the proposed system. Section 3 describes the training model and the parameter setups. Experimental results of air temperature and soil moisture forecasts are compared in Sect. 4, and related curves are drawn for analysis. Finally, Sect. 5 concludes the paper and discusses future work.

\section{System Architecture}

Figure 1 shows the proposed real-time monitoring system installed in a durian farm in Thailand with an area of $1800 \mathrm{~m}^{2}$ allocated to durian trees. We installed one set of sensors in the middle of the farm to prevent them from interfering with the farmer's work. The installation steps were as follows: (1) selection of environmental sensors, (2) installation of sensors for soil monitoring, (3) construction of wireless network system, (4) connection of electric power source, and (5) development of an app for mobile devices. The environmental sensors measure air temperature and soil moisture. Raspberry Pi 3 was employed as the microcontroller for data collection and transmission. The mobile app makes it easy for farmers to be aware of their farm's status.

Figure 2 demonstrates the results provided by the app. Farmers must enter their username and password to read the real-time data collected in the farm. With the aid of this app, farmers have immediate knowledge of the state of their farm, enabling them to control the farm environment appropriately.

The system architecture is shown in Fig. 3. The environmental data including air temperature and soil moisture are set as variables and acquired by appropriate sensors. The data are
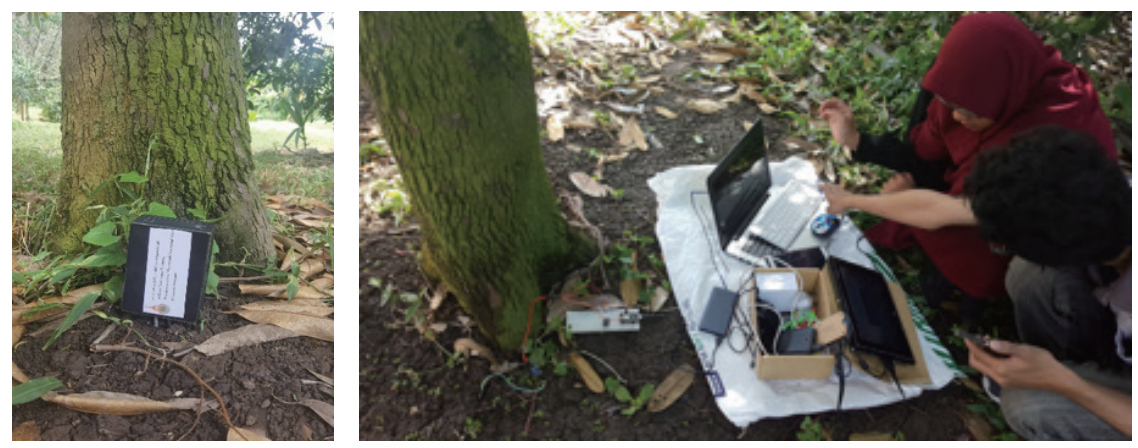

Fig. 1. (Color online) System installed in the smart durian farm. 

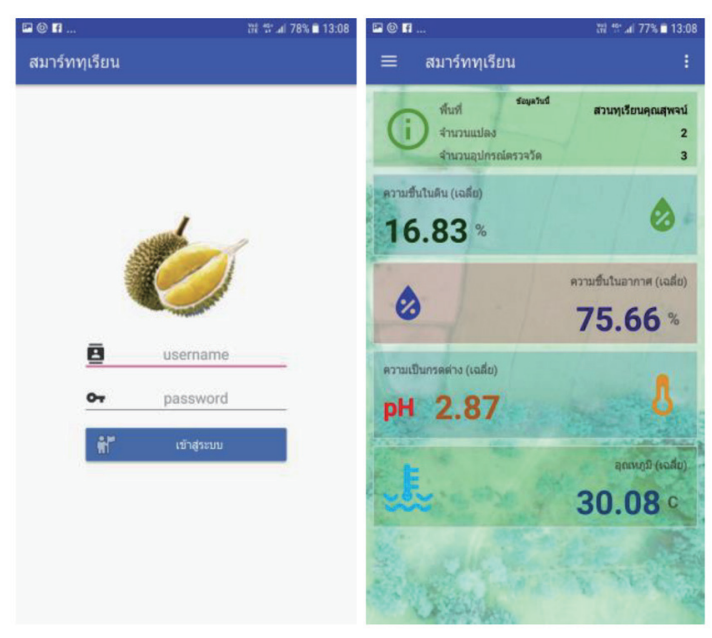

Fig. 2. (Color online) Environmental monitoring mobile app developed for durian farm.

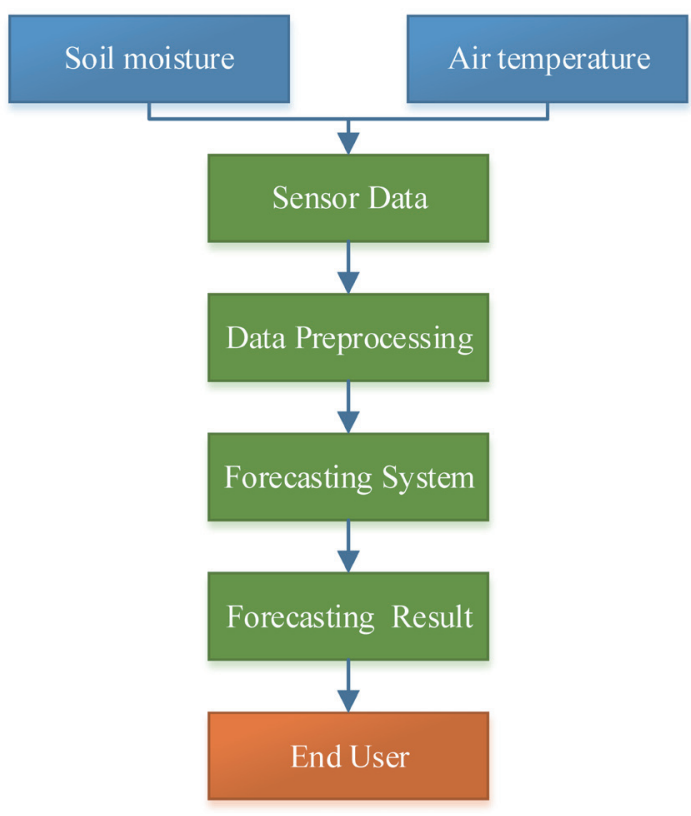

Fig. 3. (Color online) Architecture of the durian farm forecasting system.

preprocessed for cleaning and verification. Machine learning algorithms are then applied to train the preprocessed data to predict the variables. A best fit model can be found by analyzing the minimal errors. The data from the previous three days are utilized to predict data for the fourth day in both the training and testing phases. Finally, the forecasting results are presented to the end user so that appropriate measures can be followed.

\section{Proposed Model}

In this paper, we utilize some of the most popular machine learning algorithms, MLP, CNN, LSTM, SVM, and RF, for analyzing and forecasting the condition of a durian farm.

Figure 4 shows the system flowchart of the machine learning processes. The data collected from the durian farm are preprocessed for training. For each month, the first 20 days of data are used for training and the rest are used for testing. To reduce the interference of noise, an averaging filter is applied. The data input to the training network are normalized first. The training is then performed until the system converges. After training is completed, the testing data are applied to evaluate the forecasting performance.

MLP, CNN, and LSTM belong to neural network structures, as shown in Fig. 5. A typical neural network has an input layer, an output layer, and more than one hidden layer whenever required. Every neuron is fully connected to the neurons of its neighboring layer. The adopted MLP, which is shown in Fig. 6, consists of four dense layers, one dropout layer, and another dense layer for the output. The neurons in the dense layer are fully connected. The number of units in the first layer is 540, which is the average number of data points acquired over 3 days. 


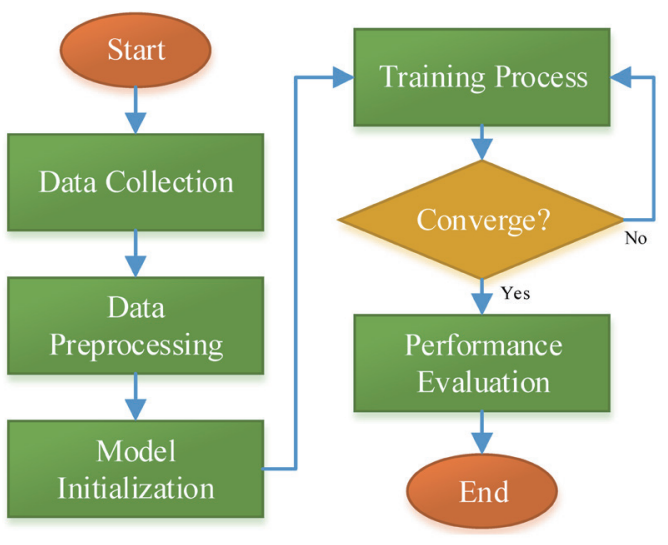

Fig. 4. (Color online) System flowchart of the training process.

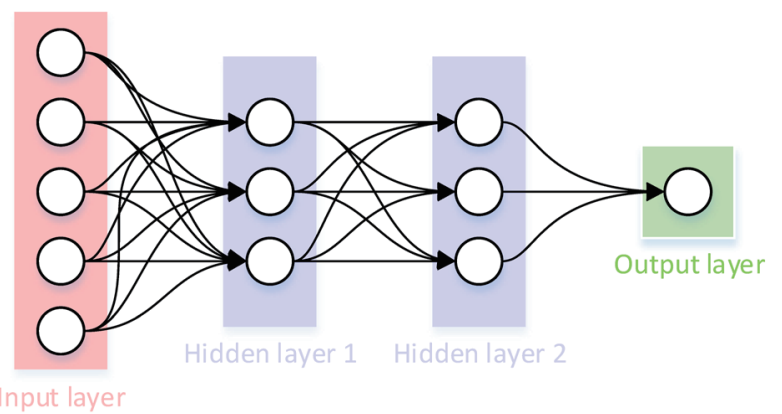

Fig. 5. (Color online) Structure of basic neural network.

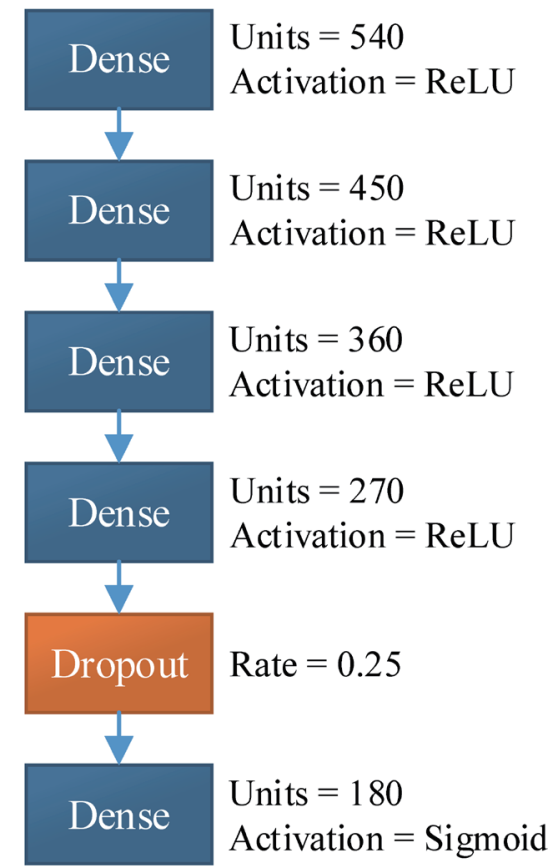

Fig. 6. (Color online) Structure of MLP.

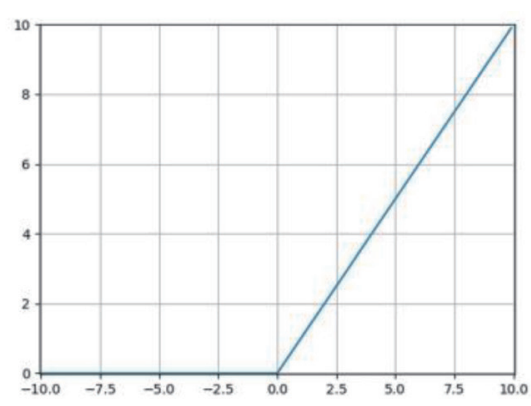

(a)

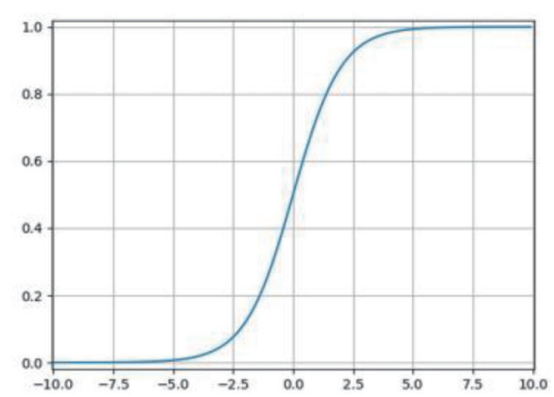

(b)

Fig. 7. (Color online) Activation functions. (a) ReLU and (b) sigmoid.

The number of units for the subsequent dense layers is decreased by 90 at each level to 450, 360, and 270 units. The utilized activation function is the rectified linear unit (ReLU). To avoid overfitting, a dropout layer with a dropout rate of 0.25 is inserted. The output layer has 180 units and uses the sigmoid activation function. The ReLU and sigmoid activation functions are shown in Fig. 7. They play an important role in preventing the gradient from vanishing during the backpropagation process and are expressed as 
ReLU: $f(x)=\max (x, 0)$,

Sigmoid: $f(x)=\frac{1}{1+e^{-x}}$.

The CNN model applied in this work is different from the CNN model used in image processing, which operates in two dimensions. Our data are time-related and only onedimensional. The full system consists of four layers of a one-dimensional CNN (Conv1D), one flattening layer, one dropout layer, and a dense layer for the output, as shown in Fig. 8. For the Conv1D layers, the kernel size is set to 9 and the activation function is ReLU. (After CNN extracts the features, a flattening layer is applied to convert the data into one dimension.) The data are then processed by a dropout layer to avoid overfitting, where the dropout rate is again 0.25 . The output dense layer has 180 processing units that use the sigmoid activation function.

LSTM is a type of recurrent neural network (RNN) and is most suitable for analyzing time series data. LSTM has the capability of capturing long-term dependences of time series, giving it better performance when making data forecasts. The RNN structure used in this study, which is shown in Fig. 9, consists of three layers of LSTM, i.e., a flattening layer, a dropout layer, and a dense output layer. Its functionality is similar to that of $\mathrm{CNN}$ as described in Fig. 8.

In addition to CNN and LSTM, other neural network structures such as SVM and RF were studied as well. SVM is a supervised learning algorithm, for which we choose the radial basis function (RBF) as the basis function. All the parameters are listed in Table 1. RF is another popular machine learning algorithm using the concept of DTs and has the advantage of

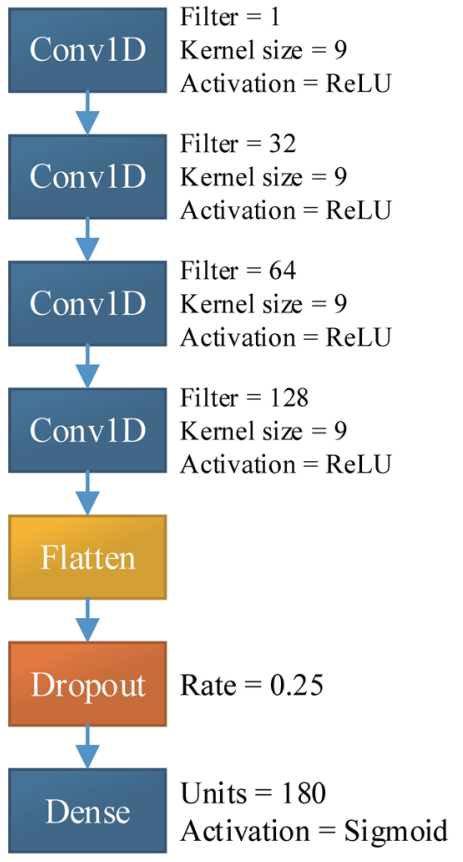

Fig. 8. (Color online) Structure of the CNN.

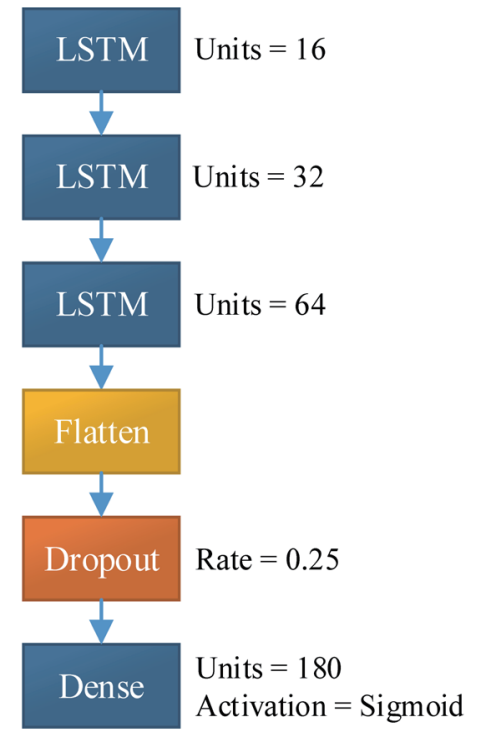

Fig. 9. (Color online) Structure of the LSTM. 
Table 1

Parameters of SVM.

\begin{tabular}{lclc}
\hline Parameter & Value or method & Parameter & Value or method \\
\hline Kernel & RBF & C & 100 \\
Degree & 3 & $\varepsilon$ & 0.1 \\
$\gamma$ & Auto & Shrinking & True \\
coef0 & 0.0 & Cache size & 200 \\
Tolerance & 1.0 & Maximum iterations & Default value \\
\hline
\end{tabular}

Table 2

Parameters of RF.

\begin{tabular}{|c|c|c|c|}
\hline Parameter & Value or method & Parameter & Value or method \\
\hline Number of estimators & 100 & Minimum samples leaf & 1 \\
\hline Criterion & MSE & Minimum weighted fraction leaf & 0.0 \\
\hline Maximum depth & 2 & Minimum impurity decrease & 0.0 \\
\hline Minimum samples split & 2 & Cost-complexity pruning alpha & 0.0 \\
\hline
\end{tabular}

simplicity. The parameters of RF used in this work are listed in Table 2. Every machine learning algorithm has advantages and disadvantages. We compare the results of applying the different algorithms to the durian farm data in the next section.

\section{Experimental Results}

To fully investigate the effectiveness of training and testing data from a durian farm, we evaluate various statistics and plots in this section. We also discuss and compare the results and performance of each algorithm.

\subsection{Data description}

The data of air temperature and soil moisture were acquired in a Thailand durian farm from July 2018 to March 2019. An averaging filter was first applied to the data to alleviate the effects of noise and interference. The method of averaging is defined as

$$
\text { Average filter }=\frac{1}{n} \sum_{i=1}^{n} x_{i}
$$

where $n$ is the number of data points and $x_{i}$ is the data reading. This operation smooths the results. Figure 10 shows the data over a period of 10 days after averaging. The top plot shows the air temperature and the bottom plot shows the soil moisture. The periodicity in the temperature variation can be observed clearly. The moisture data show higher variation than the temperature data, which may have been caused by periods of rainfall and sunshine.

A normalization process was then applied to the averaged data to limit the data to between 0 and 1. Data segments were set on a monthly basis. For each month, data from the first 20 days were used for training to build the system model. The data from the remaining 10 days were 

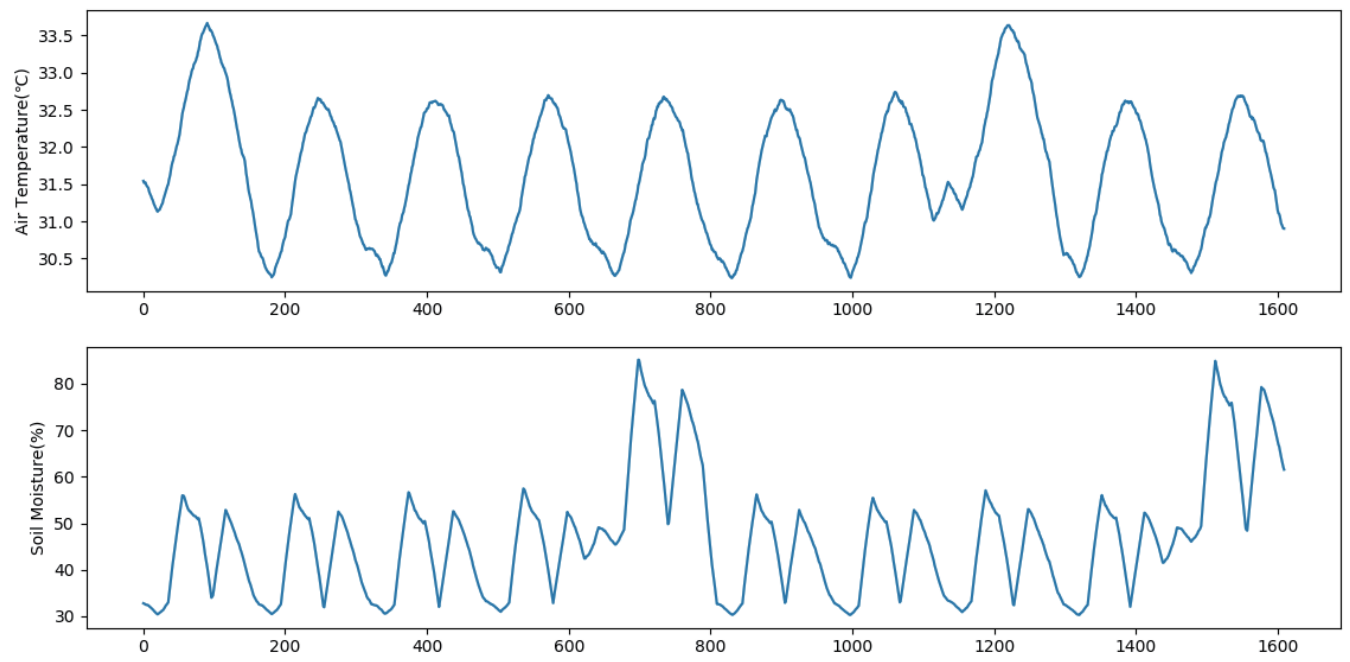

Fig. 10. (Color online) Data obtained after averaging.

used for testing and validation. The data were screened for outliers to only keep those that well represent the characteristics of the durian farm.

\subsection{Experimental results}

As reported in this section, various machine learning algorithms were adopted for the training data. These trained models were then applied to the testing data for verification. The prediction errors were compared to evaluate the performance and find the best fit model. The evaluation was performed using the method of mean absolute error (MAE), which is expressed as

$$
M A E=\frac{1}{n} \sum_{i=1}^{n}\left|x_{i}-y_{i}\right|=\frac{1}{n} \sum_{i=1}^{n}\left|e_{i}\right|
$$

where $n$ is the number of data, $x_{i}$ is the predicted value, $y_{i}$ is the actual value, and $\left|e_{i}\right|$ is the absolute error. The evaluation results of predicting air temperature and soil moisture are listed in Tables 3 and 4, respectively.

In Table 3, the rows represent different learning algorithms, the columns represent the testing trial number sorted by month, and the last column contains the average values. From the average values, we can see that SVM has the best performance, followed by CNN, LSTM, RF, and MLP. Observing the difference between the highest and lowest MAE, it is apparent that LSTM has the highest variation, which means that its performance is not very consistent. RF has the lowest variation, but its average MAE is not the lowest despite its stable performance. After discarding the worst result for each algorithm, the average MAEs for MLP, CNN, LSTM, SVM, and RF are $0.262,0.227,0.221,0.204$, and 0.288 , respectively. Again, SVM shows the best performance and consistency and CNN has the worst performance. Therefore, SVM is recommended for predicting air temperature in the durian farm. 
Table 3

Forecasting results of air temperature in terms of MAE.

\begin{tabular}{lllllllllll}
\hline & $\# 1$ & $\# 2$ & $\# 3$ & $\# 4$ & $\# 5$ & $\# 6$ & $\# 7$ & $\# 8$ & $\# 9$ & Avg. \\
\hline MLP & 0.31 & 0.22 & 0.23 & 0.21 & 0.34 & 0.19 & 0.27 & 0.23 & 0.36 & 0.327 \\
CNN & 0.24 & 0.21 & 0.17 & 0.19 & 0.34 & 0.18 & 0.24 & 0.14 & 0.33 & 0.297 \\
LSTM & 0.29 & 0.18 & 0.17 & 0.19 & 0.33 & 0.19 & 0.20 & 0.13 & 0.31 & 0.303 \\
SVM & 0.24 & 0.16 & 0.15 & 0.18 & 0.28 & 0.21 & 0.19 & 0.16 & 0.27 & 0.283 \\
RF & 0.34 & 0.28 & 0.21 & 0.24 & 0.35 & 0.25 & 0.32 & 0.21 & 0.39 & 0.325 \\
\hline
\end{tabular}

Table 4

Forecasting results of soil moisture in terms of MAE.

\begin{tabular}{lcccccccccc}
\hline & $\# 1$ & $\# 2$ & $\# 3$ & $\# 4$ & $\# 5$ & $\# 6$ & $\# 7$ & $\# 8$ & $\# 9$ & Avg. \\
\hline MLP & 9.63 & 8.44 & 4.81 & 6.12 & 7.64 & 2.20 & 2.46 & 1.99 & 3.67 & 5.743 \\
CNN & 9.38 & 7.62 & 3.08 & 2.75 & 5.00 & 1.91 & 2.10 & 1.71 & 3.43 & 4.890 \\
LSTM & 9.71 & 7.70 & 2.81 & 3.27 & 5.02 & 1.97 & 2.21 & 1.76 & 3.92 & 5.087 \\
SVM & 10.92 & 8.24 & 5.98 & 6.34 & 6.21 & 1.99 & 1.91 & 1.51 & 6.17 & 6.553 \\
RF & 9.82 & 8.22 & 4.81 & 4.33 & 4.61 & 2.61 & 2.18 & 2.00 & 3.76 & 5.302 \\
\hline
\end{tabular}

For soil moisture prediction, shown in Table 4, CNN yields the best results, followed by LSTM, RF, MLP, and SVM. We can see that the moisture data have more variation and less consistency than the temperature data. The structure of CNN has fewer constraints on timerelated data than the other algorithms, hence it gives better results than the other algorithms. We thus recommend using $\mathrm{CNN}$ for predicting soil moisture data in the durian farm.

To clearly demonstrate the effectiveness of the predictions, Fig. 11 shows plots of predicted temperature for six different time segments obtained using SVM and Fig. 12 shows plots of predicted soil moisture obtained using $\mathrm{CNN}$. Blue lines are real data, and red lines are the predicted data. The plots for the other algorithms are given in the Appendix.

Figures 13 and 14 respectively show the results of temperature and moisture prediction using various algorithms, where blue lines represent real data and the results for other algorithms are

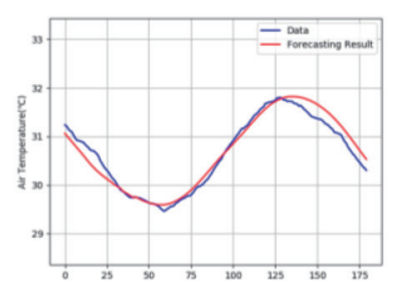

(a)

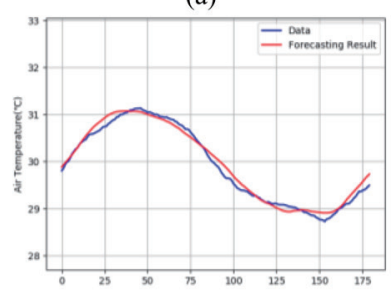

(d)

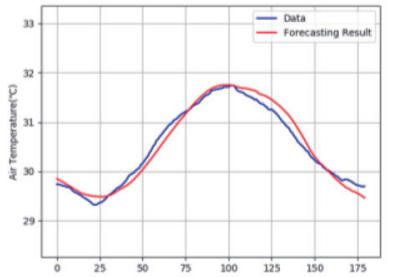

(b)

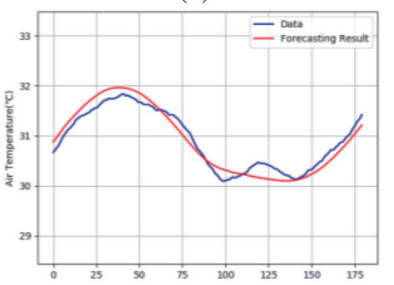

(e)

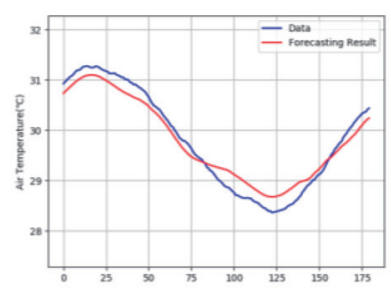

(c)

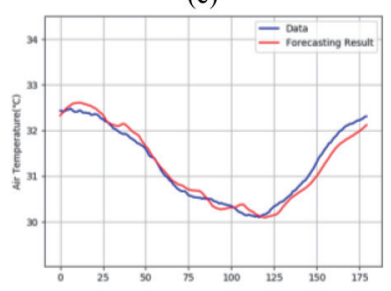

(f)

Fig. 11. (Color online) Air temperature forecasting results obtained using SVM. 


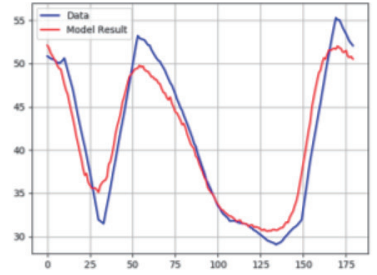

(a)

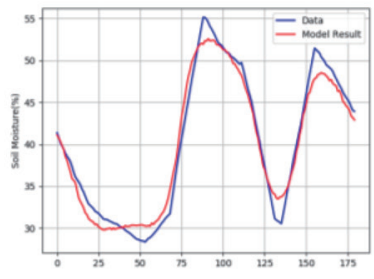

(d)

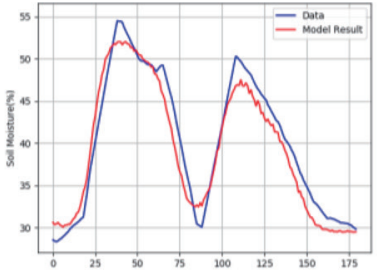

(b)

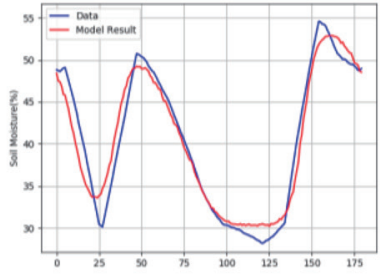

(e)

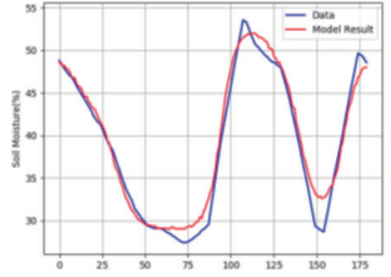

(c)

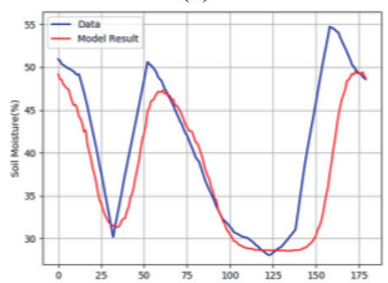

(f)

Fig. 12. (Color online) Soil moisture forecasting results obtained using CNN.

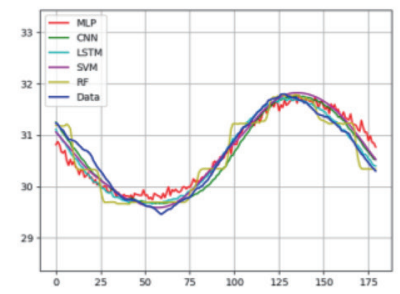

(a)

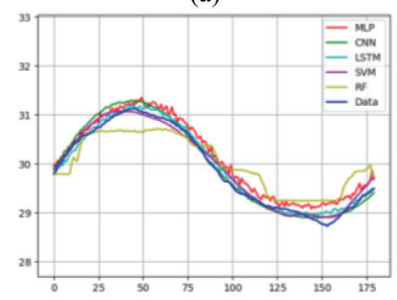

(d)

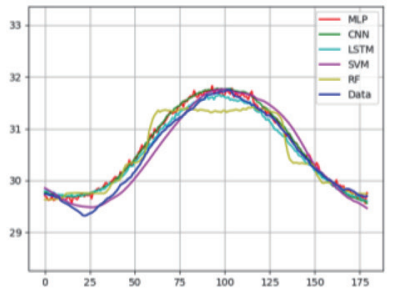

(b)

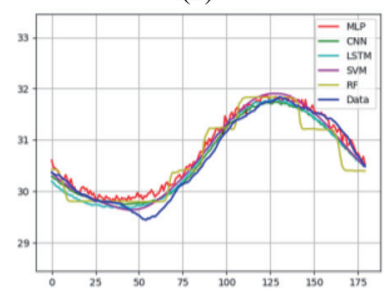

(e)

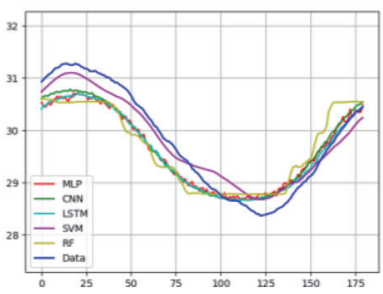

(c)

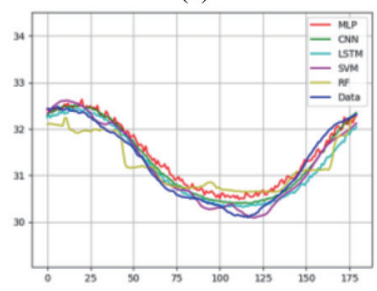

(f)

Fig. 13. (Color online) Air temperature predicted by different algorithms.

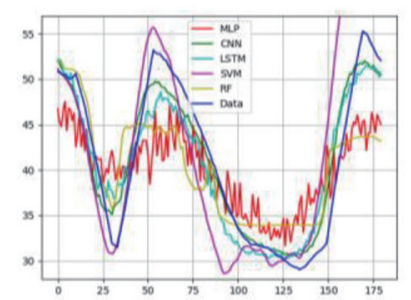

(a)

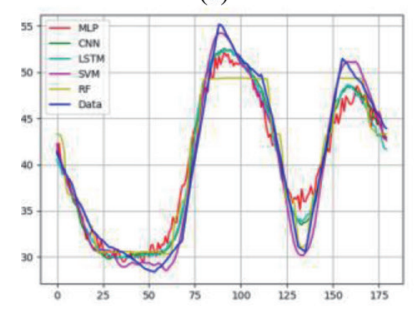

(d)

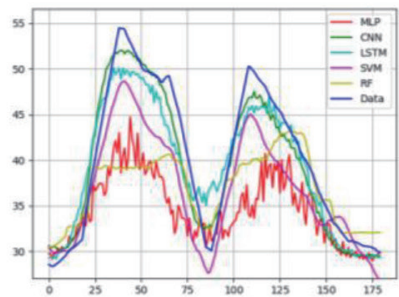

(b)

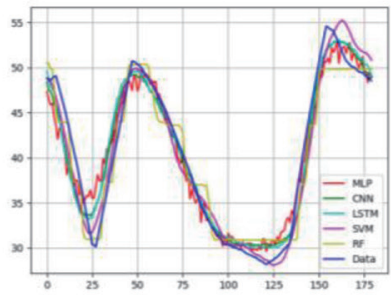

(e)

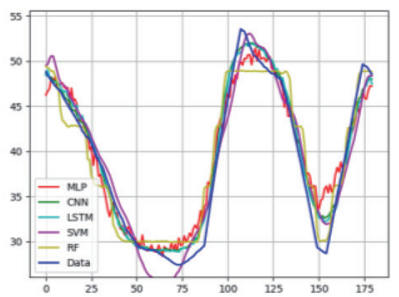

(c)

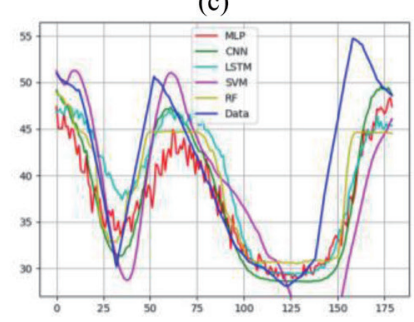

(f)

Fig. 14. (Color online) Soil moisture predicted by different algorithms. 
shown in different colors; the closer the line is to the blue line, the better the performance. The orders of performance are consistent with those in Tables 3 and 4. We also find that the prediction for soil moisture has higher errors and variations than those for temperature. This situation can be observed from the red curves corresponding to the MLP algorithm. The line belong to CNN is smoothest and closest to the blue one. LSTM shows some noise and deviates from the real data. SVM demonstrates good performance for temperature data but higher deviation for moisture data. RF appears to produce staircase-like curves, which may be due to the characteristics of DTs. Hence, the values at the peaks cannot be well approximated.

\section{Conclusions}

This paper presented machine learning algorithms for monitoring and forecasting the environmental variables of a durian farm to help maintain stable farming conditions. The variables are predicted from historical data so that farmers can take advance prevention measures. Several neural network approaches were compared and the experimental results show that SVM has the best performance for air temperature data while CNN yields the highest accuracy for soil moisture data. The results of this paper can be applied to other crops to promote intelligent agriculture techniques.

\section{Acknowledgments}

This work was supported by the Ministry of Science and Technology, Taiwan (Grants MOST 110-2221-E-153-008 and MOST 109-2221-E-194-053-MY3) and National Pingtung University, Taiwan (Grant NPTU-109-004).

\section{References}

1 J. Muangprathub, N. Boonnam, S. Kajornkasirat, N. Lekbangpong, A. Wanichsombat, and P. Nillaor: Comput. Electron. Agric. 156 (2019) 467. https://doi.org/10.1016/j.compag.2018.12.011

2 S. Sadowski and P. Spachos: Comput. Electron. Agric. 172 (2020) 105338. https://doi.org/10.1016/j. compag.2020.105338

3 D. Bandur, B. Jaksic, M. Bandur, and S. Jovic: Comput. Electron. Agric. 156 (2019) 500. https://doi. org/10.1016/j.compag.2018.12.016

4 A. B. B. Torres, A. R. da Rocha, T. L. C. da Silva, J. N. de Souza, and R. S. Gondim: Comput. Electron. Agric. 171 (2020) 105309. https://doi.org/10.1016/j.compag.2020.105309

5 J. Chen and A. Yang: IEEE Access 7 (2019) 77134. https: //doi.org/10.1109/ACCESS.2019.2921391

6 N. Ahmed, D. De, and I. Hussain: IEEE Internet Things J. 5 (2018) 4890. https://doi.org/10.1109/ JIOT.2018.2879579

7 Z. Hu, L. Xu, L. Cao, S. Liu, Z. Luo, J. Wang, X. Li, and L. Wang: IEEE Access 7 (2019) 87582. https://doi. org/10.1109/ACCESS.2019.2924917

8 A. W. Malik, A. U. Rahman, T. Qayyum, and S. D. Ravana: IEEE Internet Things J. 7 (2020) 3300. https://doi. org/10.1109/JIOT.2020.2967405.

9 G. J. Horng, M. X. Liu, and C. C. Chen: IEEE Sens. J. 20 (2020) 2766. https://doi.org/10.1109/ JSEN.2019.2954287

10 I. Sa, Z. Chen, M. Popovic, R. Khanna, F. Liebisch, J. Nieto, and R. Siegwart: IEEE Robot. Autom. Lett. 3 (2018) 588. https://doi.org/10.1109/LRA.2017.2774979

11 W. Guo, Y. Yang, Y. Zhou, Y. Tan, H. Wei, A. Song, and G. Pang: IEEE Access 6 (2018) 60214. https://doi. org/10.1109/ACCESS.2018.2873811 
12 T.-L. Lin, H.-W. Tseng, Y. Wen, F.-W. Lai, C.-H. Lin, and C.-J. Wang: IEEE Sens. J. 18 (2018) 9792. https://doi. org/10.1109/JSEN.2018.2865916

13 T. Moon, S. Hong, H. Y. Choi, D. H. Jung, S. H. Chang, and J. E. Son: Comput. Electron. Agric. 166 (2019) 105023. https://doi.org/10.1016/j.compag.2019.105023

14 J. Li, Z.-Z. Chen, L. Huang, M. Fang, B. Li, X. Fu, H. Wang, and Q. Zhao: IEEE Internet Things J. 6 (2019) 1394. https://doi.org/10.1109/JIOT.2018.2845128

15 D. Yang, X. Gao, L. Kong, Y. Pang, and B. Zhou: IEEE Trans. Consum. Electron. 66 (2020) 173. https://doi. org/10.1109/TCE.2020.2977964

16 J. Zhang, Q. Ma, X. Cui, H. Guo, K. Wang, and D. Zhu: Comput. Electron. Agric. 175 (2020) 105525. https:// doi.org/10.1016/j.compag.2020.105525

17 S. Dai, L. Li, and Z. Li: IEEE Access 7 (2019) 38287. https://doi.org/10.1109/ACCESS.2019.2907000

18 H. Li, A. Shrestha, H. Heidari, J. Le Kernec, and F. Fioranelli: IEEE Sens. J. 20 (2020) 1191. https://doi. org/10.1109/JSEN.2019.2946095

19 Y. Peng, N. Kondo, T. Fujiura, T. Suzuki, Wulandari, H. Yoshioka, and E. Itoyama: Comput. Electron. Agric. 157 (2019) 247. https://doi.org/10.1016/j.compag.2018.12.023

20 Y. Peng, N. Kondo, T. Fujiura, T. Suzuki, S. Ouma, Wulandari, H. Yoshioka, and E. Itoyama: Comput. Electron. Agric. 169 (2020) 105178. https://doi.org/10.1016/j.compag.2019.105178

21 S. Chen, J. Cao, and Z. Huang: IEEE Access 7 (2019) 57349. https://doi.org/10.1109/ACCESS.2019.2914022

22 K. Kim, C. Oh, and K. Sohn: IEEE Trans. Consum. Electron. 62 (2016) 150. https://doi.org/10.1109/ ICCE.2016.7430551

23 M. S. Oh, Z. Chen, E. Jahanshiri, D. Isa, and Y. W. Wong: Comput. Electron. Agric. 168 (2020) 105116. https:// doi.org/10.1016/j.compag.2019.105116

24 M. Shen, X. Tang, L. Zhu, X. Du, and M. Guizani: IEEE Internet Things J. 6 (2019) 7702. https://doi. org/10.1109/JIOT.2019.2901840

25 X. Guo, N. Ansari, L. Li, and H. Li: IEEE Internet Things J. 5 (2018) 4686. https://doi.org/10.1109/ JIOT.2018.2810601

26 M. Jeong, J. Nam, and B. C. Ko: IEEE Access 8 (2020) 60344. https://doi.org/10.1109/ACCESS.2020.2983202

27 D. Phan, N. Nguyen, P. N. Pathirana, M. Horne, L. Power, and D. Szmulewicz: IEEE Sens. J. 20 (2020) 723. https://doi.org/10.1109/JSEN.2019.2943879

28 J. You, S. A. S. van der Klein, E. Lou, and M. J. Zuidhof: Comput. Electron. Agric. 175 (2020) 105526. https:// doi.org/10.1016/j.compag.2020.105526

\section{Appendix}

In addition to the performance of the two algorithms with the highest performance in the main text, the performance of the other algorithms is provided in this Appendix. Figures A1-A4 show the results for air temperature using MLP, CNN, LSTM, and RF and Figs. A5-A8 show the results for soil moisture using MLP, LSTM, SVM, and RF, respectively. Again, blue lines are real data and red lines are the predicted data. 


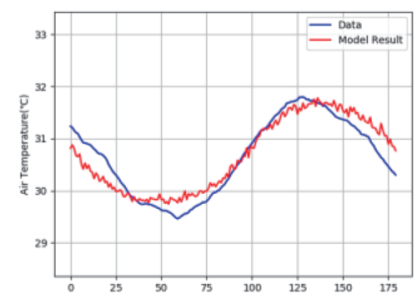

(a)

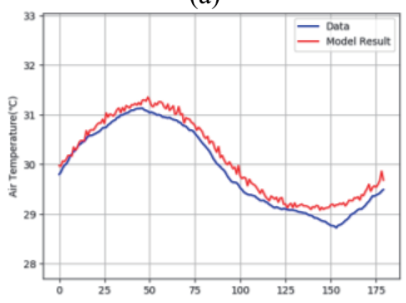

(d)

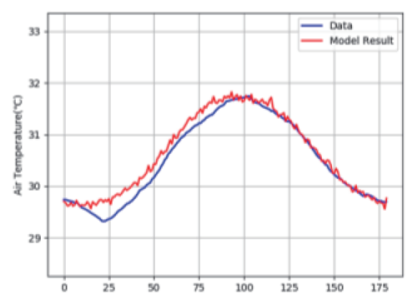

(b)

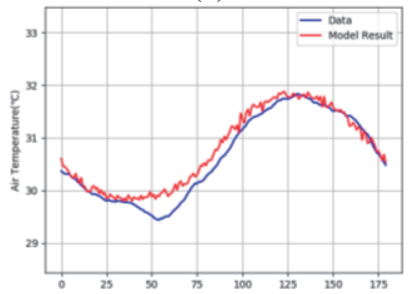

(e)

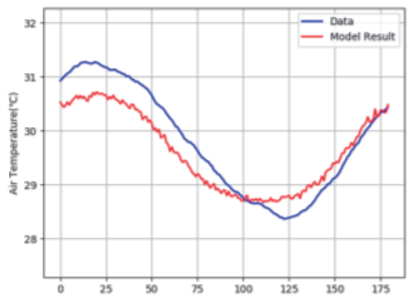

(c)

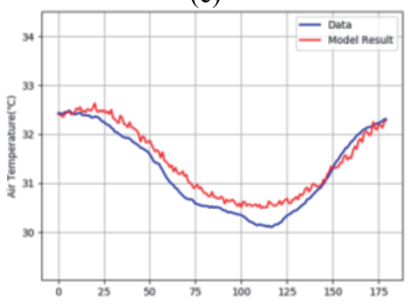

(f)

Fig. A1. (Color online) Air temperature forecasting result obtained using MLP.

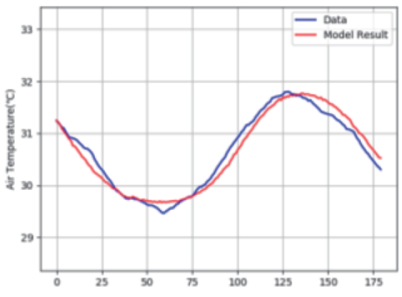

(a)

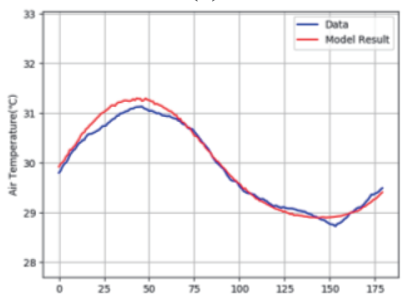

(d)

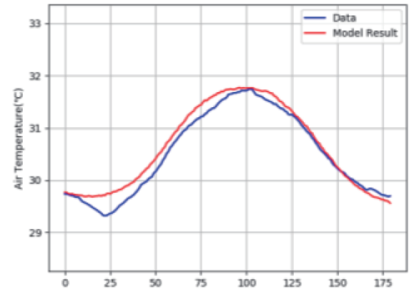

(b)

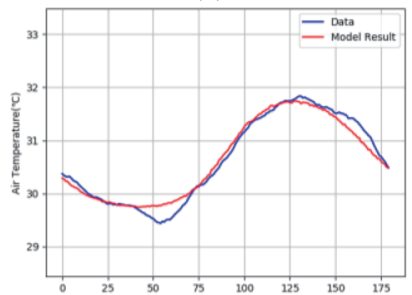

(e)

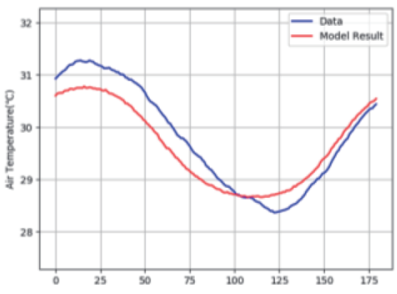

(c)

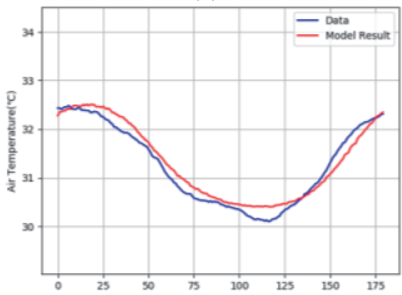

(f)

Fig. A2. (Color online) Air temperature forecasting result obtained using CNN.

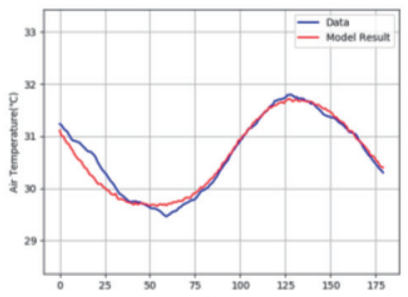

a)

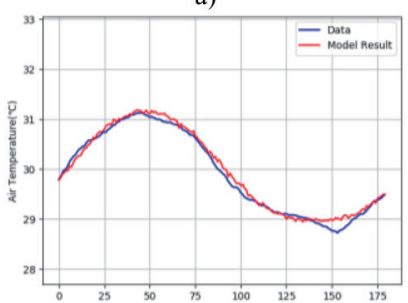

(d)

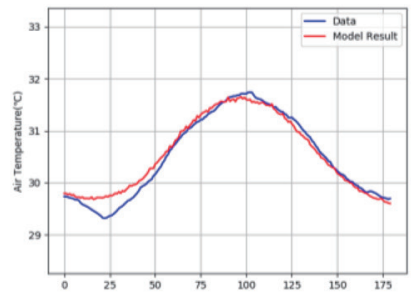

(b)

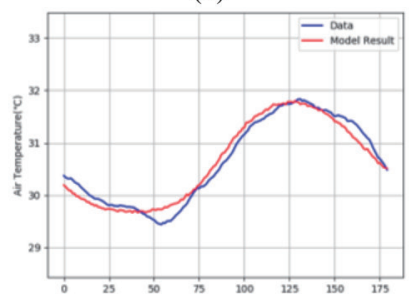

(e)

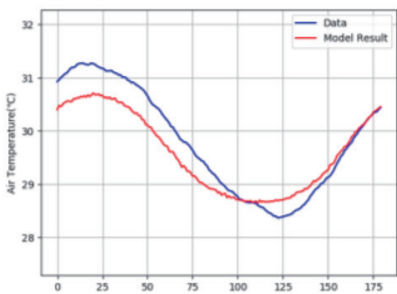

(c)

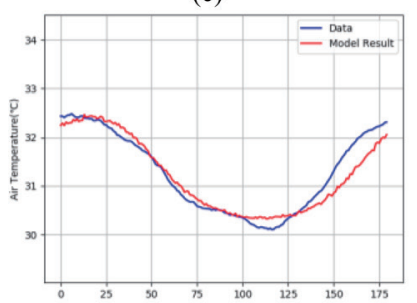

(f)

Fig. A3. (Color online) Air temperature forecasting result obtained using LSTM. 


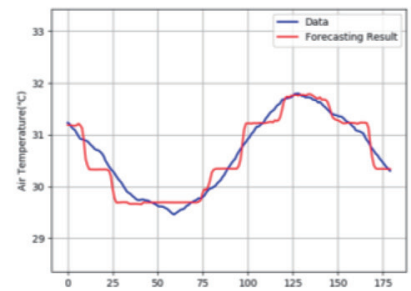

(a)

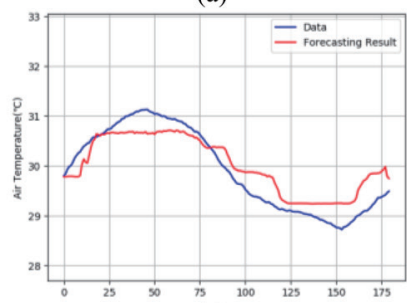

(d)

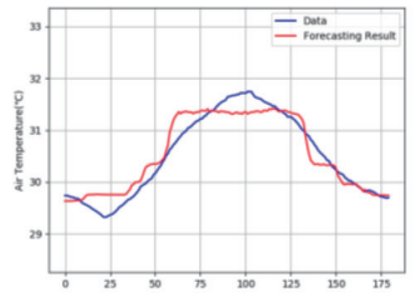

(b)

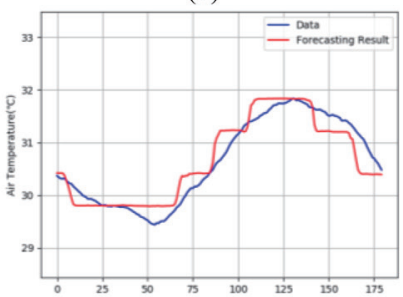

(e)

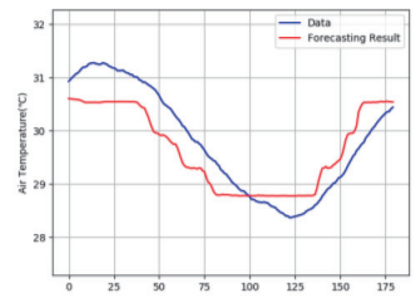

(c)

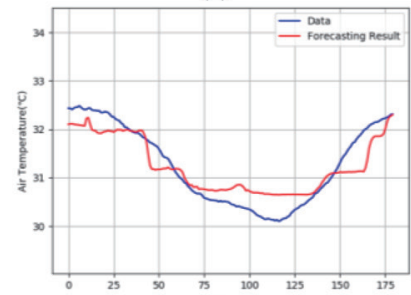

(f)

Fig. A4. (Color online) Air temperature forecasting result obtained using RF.

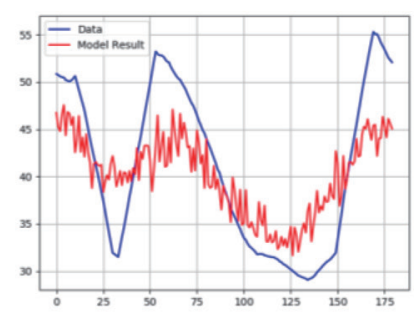

(a)

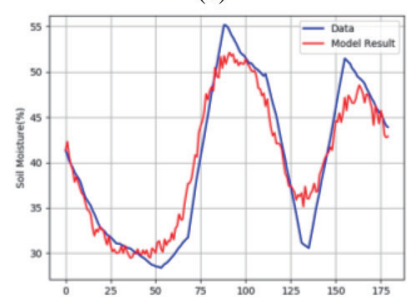

(d)

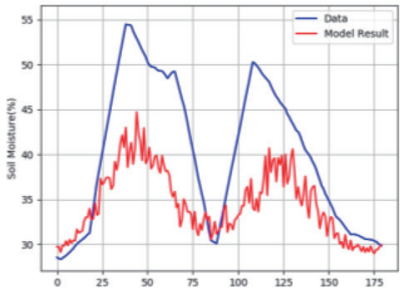

(b)

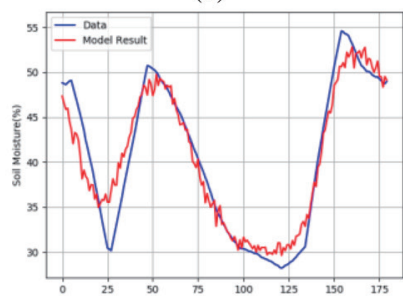

(e)

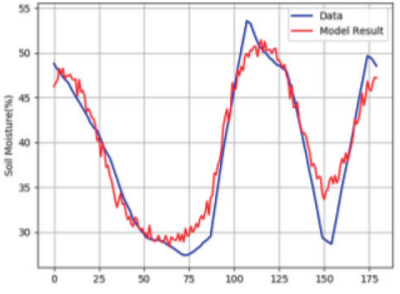

(c)

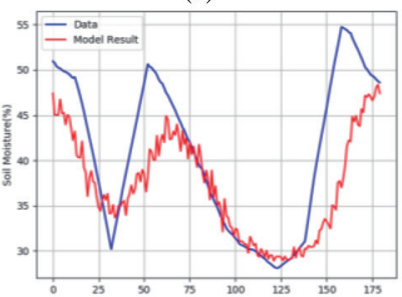

(f)

Fig. A5. (Color online) Soil moisture forecasting result obtained using MLP.

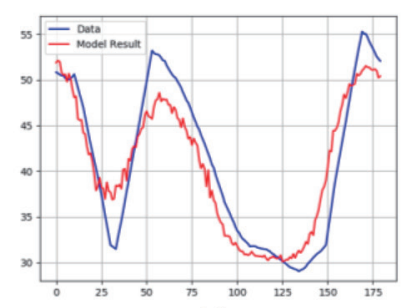

(a)

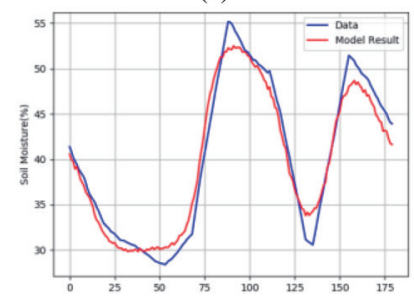

(d)

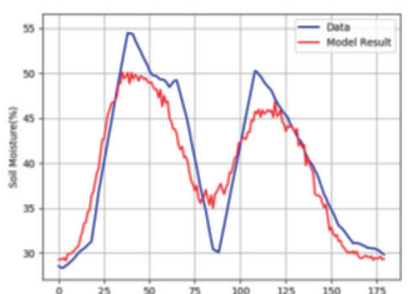

(b)

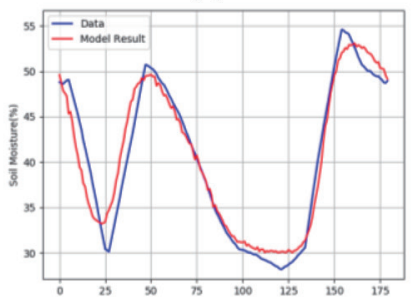

(e)

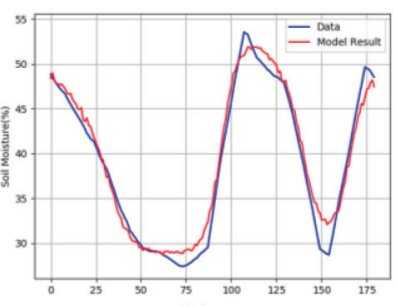

(c)

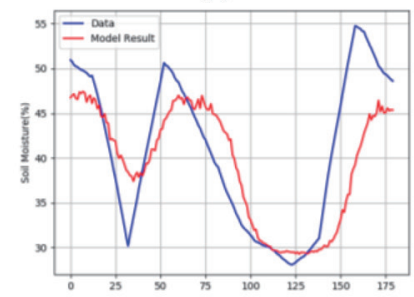

(f)

Fig. A6. (Color online) Soil moisture forecasting result obtained using LSTM. 


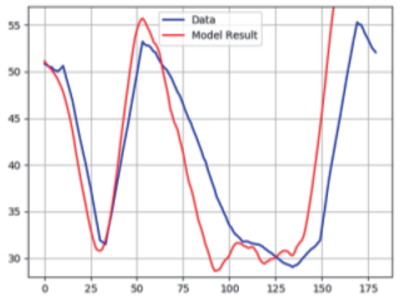

(a)

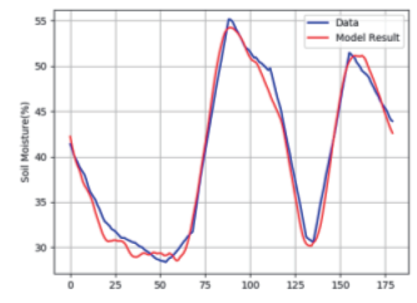

(d)

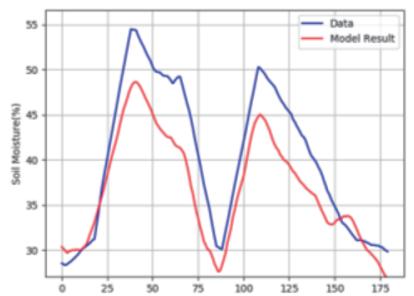

(b)

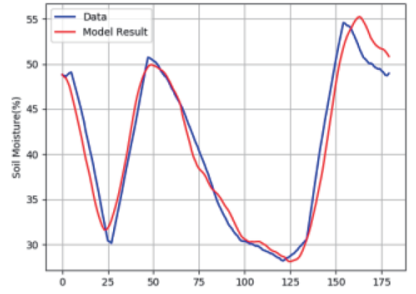

e)

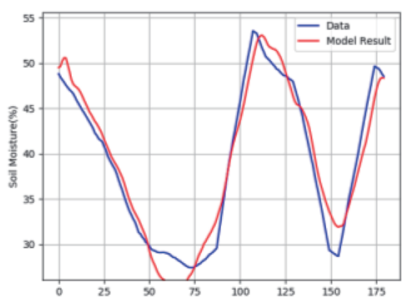

(c)

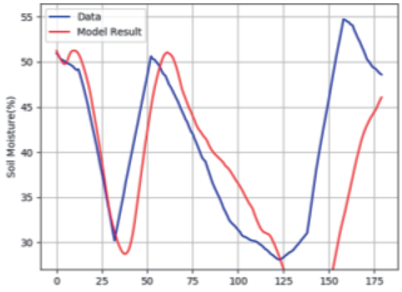

(f)

Fig. A7. (Color online) Soil moisture forecasting result obtained using SVM.

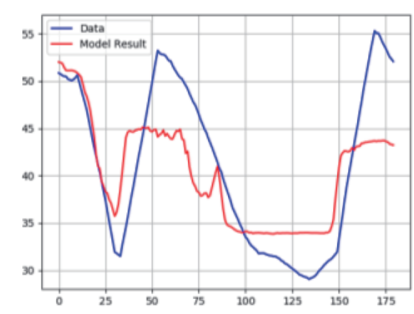

(a)

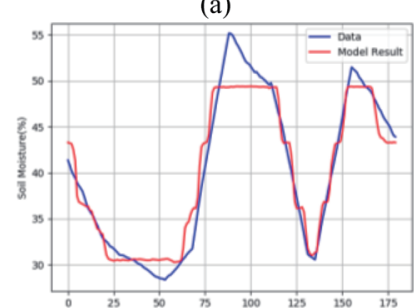

(d)

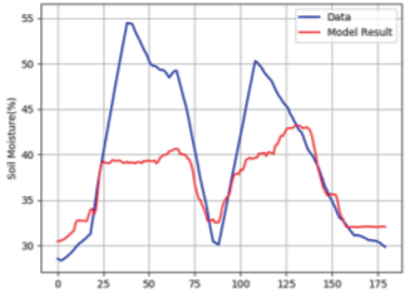

(b)

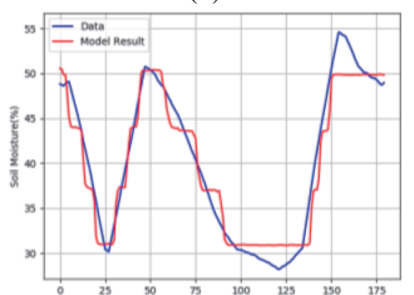

(e)

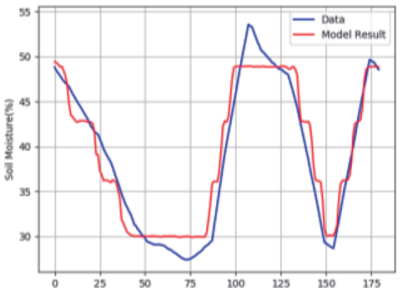

(c)

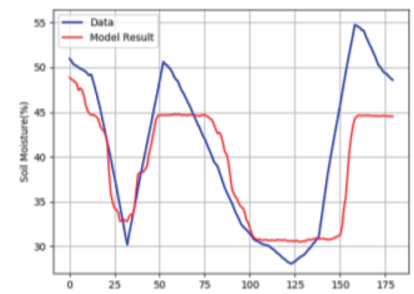

(f)

Fig. A8. (Color online) Soil moisture forecasting result obtained using RF. 